\title{
Baryonic Features in the Matter Transfer Function
}

\author{
Daniel J. Eisenstein and Wayne $\mathrm{Hu}$ \\ Institute for Advanced Study, Princeton, NJ 08540
}

\begin{abstract}
We provide scaling relations and fitting formulae for adiabatic cold dark matter cosmologies that account for all baryon effects in the matter transfer function to better than $10 \%$ in the largescale structure regime. They are based upon a physically well-motivated separation of the effects of acoustic oscillations, Compton drag, velocity overshoot, baryon infall, adiabatic damping, Silk damping, and cold-dark-matter growth suppression. We also find a simpler, more accurate, and better motivated form for the zero baryon transfer function than previous works. These descriptions are employed to quantify the amplitude and location of baryonic features in linear theory. While baryonic oscillations are prominent if the baryon fraction $\Omega_{b} / \Omega_{0} \gtrsim \Omega_{0} h^{2}+0.2$, the main effect in more conventional cosmologies is a sharp suppression in the transfer function below the sound horizon. We provide a simple but accurate description of this effect and stress that it is not well approximated by a change in the shape parameter $\Gamma$.
\end{abstract}

Subject headings: cosmology: theory - dark matter - large-scale structure of the universe

\section{Introduction}

A key success of the cold dark matter (CDM) paradigm is the ability of linear perturbation theory in the early universe to explain the power spectra observed in the cosmic microwave background (CMB) and galaxy surveys. On the largest scales, COBE finds a trend of power with scale rather close to the theoretically motivated scale-invariant spectrum (Bennett et al. 1996). On scales between 10 and $200 \mathrm{Mpc}$, however, galaxy surveys (e.g. Baugh \& Efstathiou 1993; Strauss \& Willick 1995) find a much different trend, in which the power increases with scale. Merely by including the effects of the transition between a radiation-dominated and matter-dominated universe, the CDM cosmology roughly explains both the relative normalization and the differing spectral indices of these two regimes.

Although the presence of cold dark matter does play a leading role in determining the matter power spectrum, the inclusion of baryons can lead to significant alterations. Indeed baryonic features in the power spectrum are a fundamental prediction of the gravitational instability paradigm, and their discovery would represent a strong consistency test for the cosmological model. Such features are the direct result of small density fluctuations in the early universe prior to recombination. At those times, the baryons are tightly coupled with the photons and share in the same pressure-induced oscillations that lead to acoustic peaks in the CMB. This not only leads to intermediate-scale oscillations in the power spectrum but also produces an overall suppression of power on small and intermediate scales.

\footnotetext{
${ }^{1}$ Alfred P. Sloan Fellow
} 
While the low baryon fraction $(\sim 5 \%)$ in the standard CDM model may have justified the neglect of baryonic effects on the power spectrum in the past, recent observations favor higher baryon fractions. X-ray observations of clusters of galaxies yield baryon fractions of 10-30\% (White et al. 1993; David et al. 1995). Recent measurements of high-redshift deuterium abundances (Tytler et al. 1996; but see Rugers \& Hogan 1996) and new theoretical interpretations of the Lyman- $\alpha$ forest (Weinberg et al. 1997, and references therein) suggest a value of the baryon density $\Omega_{b}$ greater than the fiducial nucleosynthesis value of $0.0125 h^{-2}$ (Walker et al. 1991). Meanwhile, observations of large-scale structure (c.f. Bahcall 1996), higher Hubble constants (c.f. Freedman 1996), and high-redshift galaxies (e.g. Spinrad et al, 1996) and clusters (Bahcall et al. 1997) favor a universe with $\Omega_{0}<1$. Such baryon fractions lead to modifications of the pure CDM transfer function that are within observational sensitivities Tegmark 1997; Goldberg \& Strauss 1997).

Numerical codes to solve the multi-species Boltzmann equations (e.g. Bond \& Efstathiou 1984; Holtzmann 1989; Hu et al. 1995; Seljak \& Zaldarriaga 1996) now agree to 1\% accuracy and run in a few minutes on today's workstations. While these codes (e.g. the publically available CMBfast) should be used for applications demanding high accuracy, analytic descriptions are useful for understanding how the different physical effects give rise to the behavior seen in the transfer function. Such descriptions better isolate the unique and robust observational signatures of physical processes in the early universe and quantify their scalings with cosmological parameters. They also probe possible parameter degeneracies and suggest possible consistency tests with related effects in the CMB.

To this end, we develop here a fitting formula for the matter transfer function of the general CDM plus baryon universe [c.f. eqs. (16)-(24) and §2]. The formula is composed of a number of well-motivated ingredients, whose behaviors we discuss in detail. We achieve fractional accuracies of $10 \%$ in fully-baryonic models and $\sim 5 \%$ in partial baryon models. Included here is a quantification of the fundamental scales including the acoustic and Silk damping scales which are related but not equal to the equivalent scales in the CMB.

We then use this form to produce quantitative assessments of the amplitude and location of baryonic oscillations as well as the alteration to the intermediate-scale shape and small-scale normalization of the transfer function. Previous assessments of the latter effects (see e.g. Peacock \& Dodd\$ 1994; Sugiyama 1995), though sufficient for the current generation of experiments at low baryon fraction, are not accurate enough for future high-precision tests expected of the Sloan Digital Sky Survey (Gunn \& Weinberg 1995) and 2-Degree Field survey (Taylor 1995). We present an approximate form that neglects the acoustic oscillations but accurately represents the suppression of power on intermediate scales [c.f. eqs. (26) and (28)-(31)]. To this end, we also present a simpler and more accurate formula [eq. (29)] for the zero-baryon transfer function (e.g. Bardeen et al. 1986).

In $\S 2$, we lay the groundwork for the subsequent discussions by presenting a summary of the physical scales that enter linear perturbation theory and the exact small-scale solution (Hu \& Sugiyama 1996, hereafter HS96) that we use to anchor our fitting formula. In $\S 3$, we state the fitting formula and discuss its performance. In $\S 4$, we describe the phenomenology revealed by the formula and present simple scalings to characterize the baryon oscillations and shape alteration. We conclude in $\S 5$. In Appendix A, we give a short guide to help the reader locate relevant formulae from the paper and turn them into COBE-normalized power spectra. A list of symbols used in this paper is given in Table 1 . 


\begin{tabular}{|c|c|c|}
\hline Symbol & Description & Equation \\
\hline$\overline{\Gamma_{0}}$ & $\Omega_{0} h$ & \\
\hline$\Gamma_{\text {eff }}$ & Effective shape & \\
\hline$\Theta_{2.7}$ & Temperature of CMB & \\
\hline$\alpha_{\Gamma}$ & $\Gamma$ suppression & \\
\hline$\alpha_{b}$ & Baryon suppression & \\
\hline$\alpha_{c}$ & CDM suppression & \\
\hline$\beta_{b}$ & Baryon envelope shift & \\
\hline$\beta_{c}$ & Shift in CDM log & \\
\hline$\beta_{\text {node }}$ & Node shift parameter & \\
\hline$R$ & Photon-baryon ratio & \\
\hline$R_{d}$ & $R$ at $z_{d}$ & \\
\hline$T$ & Transfer function (TF) & (16) \\
\hline$T_{b}$ & Baryon sector TF & 21 \\
\hline$T_{c}$ & CDM sector TF & 17 \\
\hline$T_{0}$ & Zero baryon $\mathrm{TF}$ & \\
\hline$\tilde{T}_{0}$ & Pressureless TF & \\
\hline$k$ & Wavenumber & \\
\hline$k_{\text {eq }}$ & Equality wavenumber & \\
\hline$k_{\text {Silk }}$ & Silk wavenumber & \\
\hline$q$ & $k$ scaled with $k_{\text {eq }}$ & \\
\hline$s$ & Sound horizon at drag epoch & (6), (26) \\
\hline$\tilde{s}$ & Effective sound horizon & 2) \\
\hline$z_{d}$ & Redshift of drag epoch & (4) \\
\hline$z_{\mathrm{eq}}$ & Redshift of equality & (2) \\
\hline
\end{tabular}

Table 1: A list of symbols used in this paper.

\section{Physical Effects}

To motivate and explain the transfer function formulae in $\$ 3$, we begin with a review of some of the basic results of linear perturbation theory, starting with a summary of the physical scales that enter the theory. We then describe the exact small-scale solutions found by HS96, as they play a central role in the development of the fitting formulae and the explanation of baryon phenomenology.

The particular physical properties of the constituents of the universe, in particular their equations of state and interactions, can alter the predictions of perturbation theory. Causality, however, precludes effects at arbitrarily large scales. It is therefore usual to measure the resulting perturbations by comparing them to the amplitude they would have had were causal physics neglected. The result is the transfer function, defined ast

$$
T(k) \equiv \frac{\delta(k, z=0)}{\delta(k, z=\infty)} \frac{\delta(0, z=\infty)}{\delta(0, z=0)}
$$

where $\delta(k, z)$ is the density perturbation for wavenumber $k$ and redshift $z$. By construction, $T \rightarrow 1$ as

\footnotetext{
${ }^{2}$ in the synchronous or comoving gauge; gauge issues of are no practical concern for subhorizon scales.
} 
$k \rightarrow 0$. The power spectrum $\left\langle|\delta(\mathbf{k})|^{2}\right\rangle$ is proportional to the square of the transfer function multiplied by the initial power spectrum, most often taken to be proportional to a power law $k^{n}$ with $n \approx 1$. Strictly speaking, each species of particle has a separate transfer function; however, after recombination the baryons are essentially pressureless and quickly catch up with the cold dark matter perturbations, leaving both with the same transfer function. It is this transfer function that we study in this paper.

We consider cosmologies in which the universe is primarily composed of photons, baryons (and their accompanying electrons), massless neutrinos, and cold dark matter (CDM). Relative to the critical density, the densities today of the baryons and CDM are $\Omega_{b}$ and $\Omega_{c}$ respectively. The total matter density is then $\Omega_{0}=\Omega_{b}+\Omega_{c}$. The CMB temperature $T_{\mathrm{CMB}}$ is written as $2.7 \Theta_{2.7} \mathrm{~K}$; the best observations from the COBE FIRAS instrument are $2.728 \pm 0.004 \mathrm{~K}$ (Fixsen et al. 1996; 95\% confidence interval). We assume that the massless neutrinos contribute an energy density corresponding to three species at $(4 / 11)^{1 / 3}$ the temperature of the photons. We use a Hubble constant $H_{0}$ and define $h \equiv H_{0} /\left(100 \mathrm{~km} \mathrm{~s}^{-1} \mathrm{Mpc}^{-1}\right)$. It is important to note that for $z \gg \Omega_{0}^{-1}$ the dynamics of the fluctuations are determined solely by the matter-radiation ratio $\Omega_{0} h^{2} \Theta_{2.7}^{-4}$, the baryon-photon ratio $\Omega_{b} h^{2} \Theta_{2.7}^{-4}$, and the CMB temperature $T_{\mathrm{CMB}}$. We fix the last of these to be the $C O B E$ value and do not include variations in $\Theta_{2.7}$ in our fits. Since all effects in the transfer function are set at those early times, the resulting description should depend only on $\Omega_{0} h^{2}$ and $\Omega_{b} / \Omega_{0}$. The existence today of a non-zero cosmological constant or curvature is insignificant.

\section{1. $\quad$ Length and Time Scales}

The physics governing the evolution of perturbations in CDM-baryon universes involves three distinct length scales: the horizon size at matter-radiation equality, the sound horizon at the time of recombination, and the Silk damping length at recombination.

In the usual cosmological paradigm, non-relativistic particles (baryons, electrons, and CDM) dominate relativistic particles (photons and massless neutrinos) in density today. However, because the density of these two classes of particles scale differently in time, at an earlier time, the reverse situation held. The transition from a radiation-dominated universe to a matter-dominated one occurs roughly at

$$
z_{\text {eq }}=2.50 \times 10^{4} \Omega_{0} h^{2} \Theta_{2.7}^{-4},
$$

the redshift where the two classes have equal density. As density perturbations behave differently in a radiation-dominated universe versus a matter-dominated one due to pressure support, the scale of the particle horizon at the equality epoch $z_{\text {eq }}$,

$$
k_{\text {eq }} \equiv\left(2 \Omega_{0} H_{0}^{2} z_{\text {eq }}\right)^{1 / 2}=7.46 \times 10^{-2} \Omega_{0} h^{2} \Theta_{2.7}^{-2} \mathrm{Mpc}^{-1},
$$

is imprinted on the matter transfer function; in particular, perturbations on smaller scales are suppressed in amplitude in comparison to those on large scales. If the universe consisted only of non-interacting matter and radiation, the matter transfer function would depend on the ratio $\left(k / k_{\text {eq }}\right)$ alone.

Complications arise due to interactions between the species. Prior to the recombination of baryons and electrons, the large density of free electrons couples the baryons to the photons through Coulomb and Compton interactions so that the three species move together as a single fluid. This continues until, in the process of recombination, the rate of Compton scattering between photons and electrons becomes too low, freeing the baryons from the photons. We thus define the drag epoch $z_{d}$ as the time at which the baryons are released from the Compton drag of the photons in terms of a weighted integral over the Thomson scattering 
(a) Equality-Sound Horizon

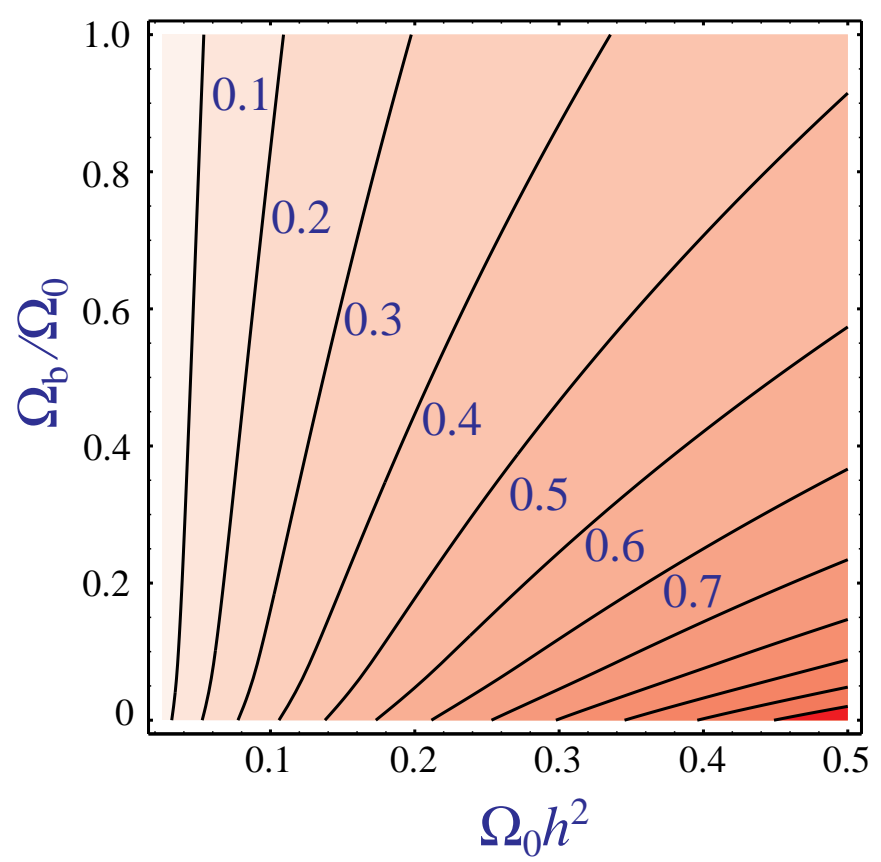

(b) Sound Horizon-Silk Scale

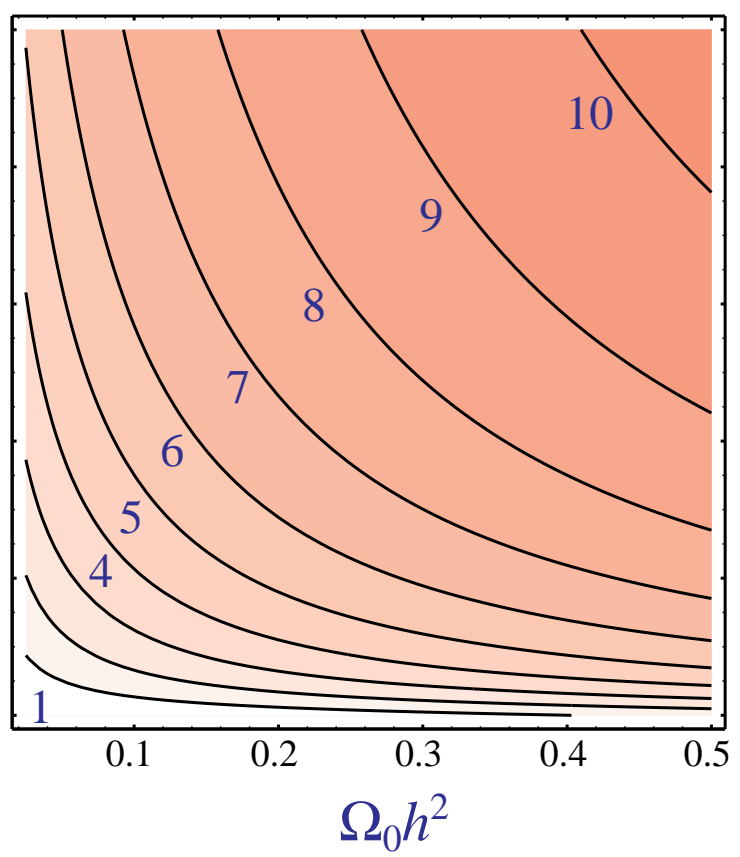

Fig. 1.- Comparison of the physical scales as functions of $\Omega_{0} h^{2}$ and the baryon fraction $\Omega_{b} / \Omega_{0}$. (a) The equality scale vs. the sound horizon: $k_{\text {eq }} s / \pi$ (unlabeled contours at 0.1 increments). (b) The sound horizon vs. the Silk scale: $k_{\mathrm{Silk}} s / \pi$ (unlabeled contours 2 and 3). The factors of $\pi$ have been included to facilitate comparison with the acoustic scale.

rate (see HS96, Eqn. C8, E2). A fit to the numerical recombination results is

$$
\begin{aligned}
z_{d} & =1291 \frac{\left(\Omega_{0} h^{2}\right)^{0.251}}{1+0.659\left(\Omega_{0} h^{2}\right)^{0.828}}\left[1+b_{1}\left(\Omega_{b} h^{2}\right)^{b_{2}}\right], \\
b_{1} & =0.313\left(\Omega_{0} h^{2}\right)^{-0.419}\left[1+0.607\left(\Omega_{0} h^{2}\right)^{0.674}\right], \\
b_{2} & =0.238\left(\Omega_{0} h^{2}\right)^{0.223}
\end{aligned}
$$

where we have reduced $z_{d}$ by a factor of 0.96 from HS96 on phenomenological grounds. For $\Omega_{b} h^{2} \lesssim 0.03$, this epoch follows last scattering of the photons.

Prior to $z_{d}$, small-scale perturbations in the photon-baryon fluid propagate as acoustic waves. The sound speed is $c_{s}=1 / \sqrt{3(1+R)}$ (in units where the speed of light is unity), where $R$ is the ratio of the baryon to photon momentum density,

$$
R \equiv 3 \rho_{b} / 4 \rho_{\gamma}=31.5 \Omega_{b} h^{2} \Theta_{2.7}^{-4}\left(z / 10^{3}\right)^{-1} .
$$

We define the sound horizon at the drag epoch as the comoving distance a wave can travel prior to redshift $z_{d}$

$$
s=\int_{0}^{t\left(z_{d}\right)} c_{s}(1+z) d t=\frac{2}{3 k_{\mathrm{eq}}} \sqrt{\frac{6}{R_{\mathrm{eq}}}} \ln \frac{\sqrt{1+R_{d}}+\sqrt{R_{d}+R_{\mathrm{eq}}}}{1+\sqrt{R_{\mathrm{eq}}}},
$$


where $R_{d} \equiv R\left(z_{d}\right)$ and $R_{\text {eq }} \equiv R\left(z_{\mathrm{eq}}\right)$ are the values of $R$ at the drag epoch and epoch of matter-radiation equality, respectively. The sound horizon at the drag epoch (hereafter simply the sound horizon) is larger than the equality horizon $\left(\sim 1 / k_{\text {eq }}\right)$ in high- $\Omega_{0}$ models but smaller than it in low- $\Omega_{0}$ models; it also decreases strongly with increasing baryon fraction if $R_{d} \gtrsim 1$ (see Fig. 1).

On small scales, the coupling between the baryons and the photons is not perfect, such that the two species are able to diffuse past one another (Silk 1968). The Silk damping scale is well fit by the approximation

$$
k_{\text {Silk }}=1.6\left(\Omega_{b} h^{2}\right)^{0.52}\left(\Omega_{0} h^{2}\right)^{0.73}\left[1+\left(10.4 \Omega_{0} h^{2}\right)^{-0.95}\right] \mathrm{Mpc}^{-1},
$$

which represents a $\pm 20 \%$ phenomenological correction from the value given in HS96. The Silk scale is generally a smaller length scale than either $s$ or $1 / k_{\text {eq }}$. Note that the difference between the drag and last scattering epochs implies that for $\Omega_{b} h^{2} \lesssim 0.03$ the sound and Silk scales in the transfer function are larger than those in the CMB.

We show a comparison of these scales as a function of cosmological parameters in Figure 1 .

\subsection{Small-scale Solutions}

In the small-scale limit, one can solve the linear perturbation equations analytically in the approximation that baryons provide no gravitational source to the CDM (HS96). This approximation is appropriate below the sound horizon since baryon perturbations are pressure supported. As we will use this solution in order to anchor the small-scale end of our fitting formulae, we describe the solutions further.

The transfer function is written as a sum of the baryon and cold dark matter contributions at the drag epoch

$$
T(k)=\frac{\Omega_{b}}{\Omega_{0}} T_{b}(k)+\frac{\Omega_{c}}{\Omega_{0}} T_{c}(k) .
$$

The CDM transfer function can be solved exactly in terms of hypergeometric functions that are more conveniently approximated by the following form:

$$
\begin{aligned}
T_{c} & \rightarrow \alpha_{c} \frac{\ln 1.8 \beta_{c} q}{14.2 q^{2}} \\
q & =\left(k / \mathrm{Mpc}^{-1}\right) \Theta_{2.7}^{2}\left(\Omega_{0} h^{2}\right)^{-1}=\frac{k}{13.41 k_{\mathrm{eq}}},
\end{aligned}
$$

where $\alpha_{c}$ and $\beta_{c}$ are fit by

$$
\begin{aligned}
\alpha_{c} & =a_{1}^{-\Omega_{b} / \Omega_{0}} a_{2}^{-\left(\Omega_{b} / \Omega_{0}\right)^{3}} \\
a_{1} & =\left(46.9 \Omega_{0} h^{2}\right)^{0.670}\left[1+\left(32.1 \Omega_{0} h^{2}\right)^{-0.532}\right], \\
a_{2} & =\left(12.0 \Omega_{0} h^{2}\right)^{0.424}\left[1+\left(45.0 \Omega_{0} h^{2}\right)^{-0.582}\right], \\
\beta_{c}^{-1} & =1+b_{1}\left[\left(\Omega_{c} / \Omega_{0}\right)^{b_{2}}-1\right], \\
b_{1} & =0.944\left[1+\left(458 \Omega_{0} h^{2}\right)^{-0.708}\right]^{-1} \\
b_{2} & =\left(0.395 \Omega_{0} h^{2}\right)^{-0.0266}
\end{aligned}
$$

As $\Omega_{b} / \Omega_{0} \rightarrow 0, \alpha_{c}, \beta_{c} \rightarrow 1$. Equation (9) shows the familiar $\ln (k) / k^{2}$ dependence of the small-scale CDM transfer function. This occurs because outside the horizon, density perturbations grow as $k^{2}$ due the product 
(a) CDM Suppression

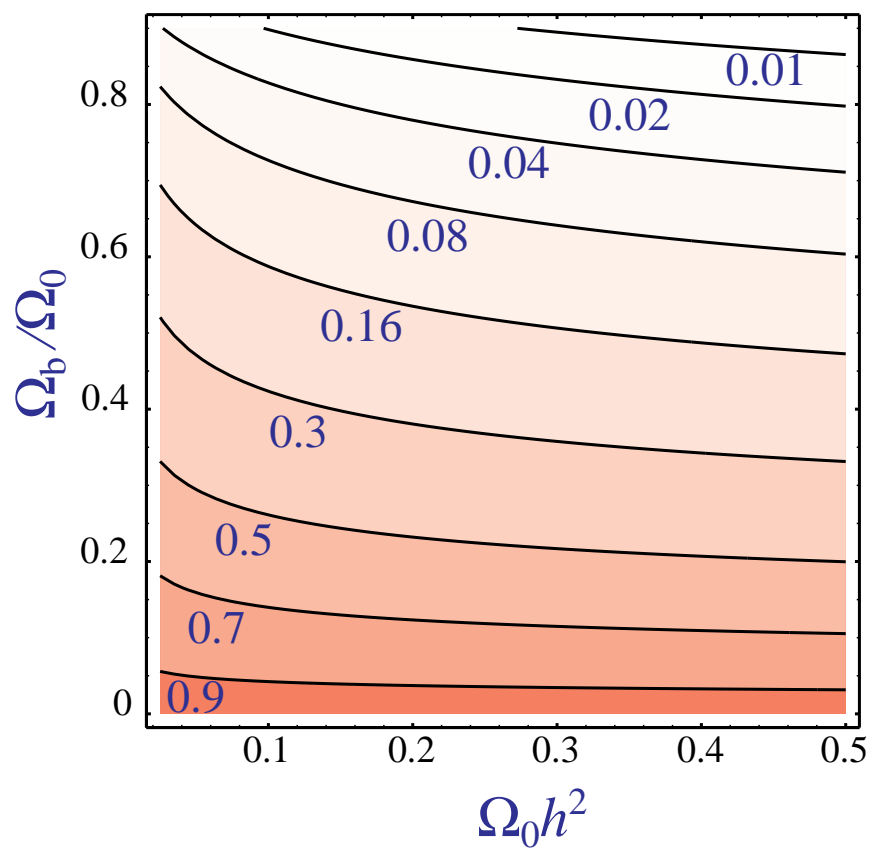

(b) Baryon Suppression

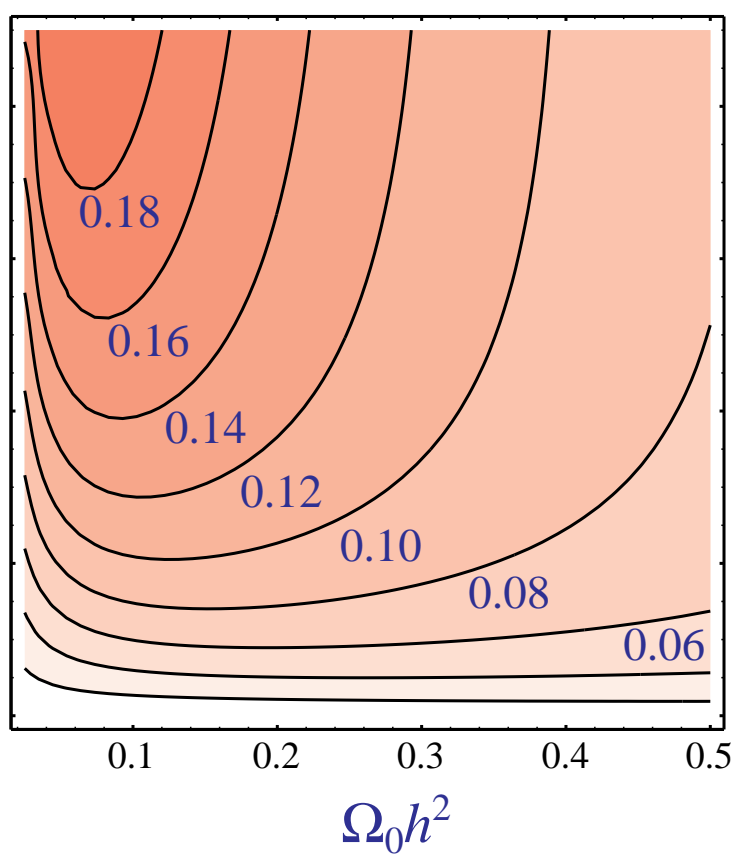

Fig. 2.- Suppression factors for (a) the $\mathrm{CDM}\left(\alpha_{c} \Omega_{c} / \Omega_{0}\right)$ and (b) the baryonic acoustic oscillations $\left(\alpha_{b} \Omega_{b} / \Omega_{0}\right)$.

of potential and velocity gradients which drive the growth; inside the horizon in the radiation dominated epoch the growth is logarithmic. The main effect of the baryons comes from the suppression in growth rates between equality and the drag epoch. As $\Omega_{0} h^{2}$ increases the time between the two epochs increases; thus the maximum suppression due to the baryons occurs in the highest $\Omega_{0} h^{2}$ models. A plot of $\alpha_{c} \Omega_{c} / \Omega_{0}$ is shown in Figure 2a.

In the small-scale limit, the baryons are trapped in acoustic oscillations until recombination permits them to slip past the photons. While the density perturbation of this oscillation contributes to the transfer function, the corresponding velocity perturbation actually dominates in the small-scale limit. When the oscillation is released at the drag epoch, the baryons move kinematically according to their velocity and generate a new density perturbation (Sunyaev \& Zel'dovich 1970; Press \& Vishniad 1980). This so-called velocity overshoot means that the transfer function for $k s \gg 1$ follows

$$
T_{b} \rightarrow \alpha_{b} \frac{\sin (k s)}{k s} \mathcal{D}(k) \text {. }
$$

Here $\mathcal{D}(k)$ represents the effects of Silk damping, which occurs due to combination of diffusion of the photons with respect to the baryons and Compton drag moving baryons from overdensities to underdensities and hence destroying the perturbation. That the dependence is $\sin (k s)$ rather than $\cos (k s)$ is the result of the dominance of the velocity term rather than the density term. A detailed treatment allows one to calculate $\alpha_{b}$ :

$$
\alpha_{b}=2.07 k_{\mathrm{eq}} s\left(1+R_{d}\right)^{-3 / 4} G\left(\frac{1+z_{\mathrm{eq}}}{1+z_{d}}\right)
$$




$$
G(y)=y\left[-6 \sqrt{1+y}+(2+3 y) \ln \left(\frac{\sqrt{1+y}+1}{\sqrt{1+y}-1}\right)\right],
$$

The factor $\left(1+R_{d}\right)^{-3 / 4}$ comes from the damping of oscillations resulting from the adiabatic decrease in the sound speed (Peebles \& Yu 1970; HS96, eq. [A17]). The factor involving $G(y)\left(\propto y^{-1 / 2}\right.$ for $\left.y \gg 1\right)$ comes from the product of the growth suppression between equality and the drag epoch $\left(\propto y^{-1}\right)$ and the time available before the velocities creating the perturbation decay due to the free expansion of the universe $\left(\propto y^{1 / 2}\right)$. A plot of $\alpha_{b} \Omega_{b} / \Omega_{0}$ is shown in Figure $2 \mathrm{~b}$.

That the phase of the oscillations is $k s$ (Peebles \& Yu 1970) can be seen simply from integrating the dispersion relation $\omega=k c_{s}$. The change in phase for an acoustic wave is $\delta \phi=[k(1+z)] c_{s} \delta t$. Integrating this from $t \approx 0$ to the drag epoch (where the baryons are released and the oscillations freeze-out) yields $k s$, owing to the definition in equation (6). A technical complication occurs for $k \gtrsim k_{\text {Silk }}$. The presence of strong damping slightly raises the redshift at which the oscillations freeze-out, making $s$ a few percent smaller (see HS96 Fig. 2). We have neglected this effect because it occurs at sufficiently small scales that the resulting phase shift is unobservable in practice, but one can see the deviations when comparing to numerical results (c.f. Fig. 3).

\section{Fitting Formulae}

As we have seen in $\$ 2$, analytic solutions exist for the transfer function at both large and small scales. The transition between these extremes is defined by two scales, the horizon at matter-radiation equality and the sound horizon at the end of the drag epoch. The former sets the dynamics of the expansion and perturbation growth; the latter sets the scale at which pressure support becomes important for the baryons. Because the range of scales accessible by the study of structure formation falls within this transition regime, it is important to understand the full transfer function in detail. To that end, we present in this section a fitting formula that approximates the full transfer function on all scales.

We write the transfer function as the sum of two pieces,

$$
T(k)=\frac{\Omega_{b}}{\Omega_{0}} T_{b}(k)+\frac{\Omega_{c}}{\Omega_{0}} T_{c}(k),
$$

whose origins lie in the evolution before the drag epoch of the baryons and cold dark matter respectively. This separation is physically reasonable as before the drag epoch the two species were dynamically independent and after the drag epoch their fluctuations are weighted by the fractional density they contribute. This automatically includes in $T_{c}$ the effects of baryonic infall into CDM potential wells. Note however that $T_{b}$ and $T_{c}$ are themselves not true transfer functions as they do not reflect the density perturbations of the relevant species today. Rather, it is their density-weighted average $T(k)$ that is the transfer function for both the baryons and the CDM.

\subsection{Cold Dark Matter}

The transfer function for cosmologies in which non-interacting cold dark matter dominates over baryons have been studied by many authors and accurate fitting formulae already exist in this limit (e.g. Bond \&

Efstathiou 1984; Bardeen et al. 1986; but see improvements in $\$$ 1.2) However the effect of baryons, though long known from numerical calculations (e.g. Peebles \& Yu 1970; Holtzmann 1989), have in the past either 
been included in fitting formulae in an ad hoc manner (see e.g. Peacock \& Dodds 1994; Sugiyama 1995) or only in the small scale limit (HS96).

In the presence of baryons, the growth of CDM perturbations is suppressed on scales below the sound horizon. The change to the asymptotic form can be calculated and has been shown in equations (9)-(12). We introduce this suppression by interpolating between two solutions near the sound horizon:

$$
\begin{aligned}
T_{c}(k) & =f \tilde{T}_{0}\left(k, 1, \beta_{c}\right)+(1-f) \tilde{T}_{0}\left(k, \alpha_{c}, \beta_{c}\right) \\
f & =\frac{1}{1+(k s / 5.4)^{4}},
\end{aligned}
$$

with

$$
\begin{aligned}
\tilde{T}_{0}\left(k, \alpha_{c}, \beta_{c}\right) & =\frac{\ln \left(e+1.8 \beta_{c} q\right)}{\ln \left(e+1.8 \beta_{c} q\right)+C q^{2}}, \\
C & =\frac{14.2}{\alpha_{c}}+\frac{386}{1+69.9 q^{1.08}} .
\end{aligned}
$$

$q, \alpha_{c}$, and $\beta_{c}$ have been given in equations (10), (11), and (12), respectively.

\subsection{Baryons}

In the case of cosmologies without cold dark matter, the transfer function departs from unity below the sound horizon to exhibit a series of declining peaks due to acoustic oscillations. The small-scale exact solution of equation (13) suggests that these may be written as the product of a declining oscillatory term, a suppression due to the decay of potentials between the equality and drag scales, and an exponential Silk damping. We therefore write

$$
T_{b}=\left[\frac{\tilde{T}_{0}(k ; 1,1)}{1+(k s / 5.2)^{2}}+\frac{\alpha_{b}}{1+\left(\beta_{b} / k s\right)^{3}} e^{-\left(k / k_{\text {Silk }}\right)^{1.4}}\right] j_{0}(k \tilde{s}) .
$$

Here the spherical Bessel function $j_{0}(x) \equiv \sin (x) / x$ is a piece that approaches unity above the sound horizon but oscillates below it. The envelope in square brackets traces the zero-baryon CDM case above the sound horizon and then breaks to a constant multiplied by an exponential Silk damping factor. We attach the Silk damping factor only to the second term because such diffusion can only occur on scales below the sound horizon, where only the second term contributes significantly. This subtlety marginally improves the fit. The sound horizon $s$, Silk scale $k_{\text {Silk }}$, and amplitude suppression $\alpha_{b}$ were given in equations (6), (7), and (14) respectively; we now discuss $\tilde{s}$ and $\beta_{b}$.

While the nodes of the baryonic transfer function asymptotically approach those of $\sin (k s)$ for $k s \gg 1$, the first few nodes $(k s \lesssim 10)$ fall at higher $k$ than predicted by $\sin (k s)$. This shift is due to the contribution of the baryon density perturbation itself at the drag epoch and reflects the fact that at the sound horizon velocity overshoot is not the dominant effect. This effect increases with $\Omega_{0} h^{2}$ because the time available for velocity overshoot (see eq. 14]) decreases as $\left(z_{d} / z_{\mathrm{eq}}\right)^{1 / 2}$ and is only weakly dependent on the baryon fraction. We have verified this explanation of the node shift by isolating the density and velocity contributions of the baryons at the drag epoch from numerical evolution codes.

We address this shifting of the nodes phenomenologically by introducing the quantity

$$
\tilde{s}(k)=\frac{s}{\left(1+\left(\beta_{\text {node }} / k s\right)^{3}\right)^{1 / 3}} .
$$


For $k s \gg \beta_{\text {node }}, \tilde{s} \rightarrow s$, restoring the sinusoidal nodes. However, at $k s \lesssim \beta_{\text {node }}, \tilde{s} \approx k s^{2} / \beta_{\text {node }}<s$, moving the nodes to higher $k$. We find

$$
\beta_{\text {node }}=8.41\left(\Omega_{0} h^{2}\right)^{0.435},
$$

independent of the baryon fraction. Hence the effect gets smaller at low $\Omega_{0}$ as expected.

The amplitude $\alpha_{b}$ specifies the small-scale asymptotic contribution of the velocity portion of the acoustic oscillation. Two effects modify this amplitude at large scales. Above the sound horizon, velocities contributions to the transfer function fall off. Furthermore, the amplitude declines if CDM dominates the energy density of the photon-baryon system when the wavelength enters the horizon. This occurs due the absence of feedback in the gravitational driving of the photon-baryon oscillator (HS96 §3.1). The net result is a cut-off associated with the sound horizon which moves to smaller scales as $\Omega_{0} h^{2}$ increases and/or $\Omega_{b} / \Omega_{0}$ decreases. We describe this in equation (21) by turning on the velocity term at the characteristic scale $\beta_{b} s$, where

$$
\beta_{b}=0.5+\frac{\Omega_{b}}{\Omega_{0}}+\left(3-2 \frac{\Omega_{b}}{\Omega_{0}}\right) \sqrt{\left(17.2 \Omega_{0} h^{2}\right)^{2}+1} .
$$

\subsection{Performance}

For the parameter range $0.025 \lesssim \Omega_{0} h^{2} \lesssim 0.25$ and $0 \leq \Omega_{b} / \Omega_{0} \leq 1$ the fitting formula works quite well. For fully baryonic models (i.e. $\Omega_{c}=0$ ) the fractional residuals are nearly always under $10 \%$. As the baryon fraction decreases, the accuracy improves due to the increasing contribution of the simpler CDM piece. Residuals smaller than $5 \%$ are typical for $\Omega_{b} / \Omega_{0}<0.5$. Note that we quote the residuals as the difference between the fit and the numerical results divided by a non-oscillatory envelope that is defined by replacing $\sin (k \tilde{s}) / k \tilde{s}$ in equation (21) by $\left[1+(k \tilde{s})^{4}\right]^{-1 / 4}$. This envelope matches the knee of the transfer function and grazes all the subsequent maxima.

In Figure 3 we display four comparisons of the fitting formula relative to the numerical results. Also shown are the residuals relative to our envelope. The reason for the degradation in the fit at the shortest scales is that small errors in the sound horizon (c.f. the end of \$2.2) produce significant errors in the phase of the oscillations, producing order unity residuals. However, the fitting formula reproduces the correct amplitude and hence the Silk scale.

The most serious systematic error in the fitting formula occurs for $\Omega_{0} h^{2} \gtrsim 0.25$. In the baryon sector of these cases, the drop between $k s \approx 1$ and the oscillations at $k s \gtrsim 5$ becomes quite precipitous. Our formula does not decline this quickly, causing the amplitude of the first valley to be significantly overestimated. Later peaks and valleys are underestimated in an attempt to compensate. One can see the beginnings of this trend in the $\Omega_{0}=\Omega_{b}=1$ example in Figure $\beta$; the problem gets more severe for higher $\Omega_{0} h^{2}$.

A less important systematic effect occurs for high baryon fraction $\left(0.7<\Omega_{b} / \Omega_{0}<0.9\right)$, low- $\Omega$ models. Due to a small shift in the CDM break scale (eq. [18]) that we have chosen not to model, the first valley is systematically underestimated by $\sim 10-15 \%$ in amplitude. This problem does not extend to lower baryon fractions.

\section{Phenomenology}

There are a number of phenomenological trends as a function of cosmological parameters. The two basic effects that arise from the inclusion of baryons are the introduction of oscillations and the suppression 



Fig. 3.- Four examples of the fit compared to numerical results. The larger plots show the numerical result (solid) and the fit (dashed). The smaller subplots show the residuals, defined as the difference between the two divided by a non-oscillatory envelope. Note that in the fully baryonic models, the oscillations have alternating sign in the transfer function. Also shown is the zero baryon case (dotted); note the strong suppression on scales below the sound horizon due to the baryons. 


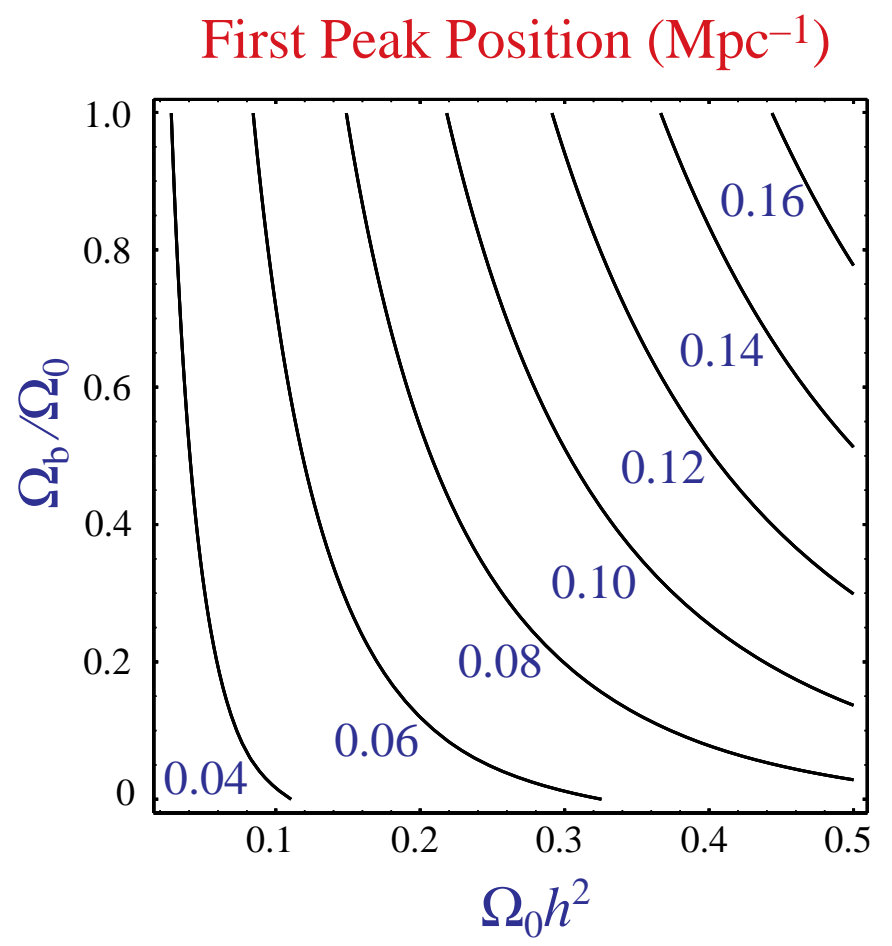

Fig. 4.- The location of the first peak in $\mathrm{Mpc}^{-1}$ as a function of cosmological parameters.

of power below the sound horizon, with a corresponding sharpening of the bend around the sound horizon. We discuss these two in turn.

\subsection{Baryon Oscillations}

Two interesting aspects of the baryonic oscillations are the location and amplitude of the peaks and troughs. Well under the sound horizon we expect them to fall at $k=m \pi / 2 s$ where $m=3,7,11 \ldots$ for troughs and $m=5,9,13 \ldots$ for peaks. However as described in $\$ 3.2$, the first few oscillations are shifted according to the parameter $\beta_{\text {node }}$. As $\Omega_{0} h^{2}$ increases, the first few peaks and troughs are progressively shifted to higher $k$. A corollary to this shift is that the ratio of the node locations becomes smaller as one raises $\Omega_{0}$, i.e. the valleys and peaks become slightly narrower. The location of the first peak is conveniently fit as

$$
k_{\text {peak }}=\frac{5 \pi}{2 s}\left(1+0.217 \Omega_{0} h^{2}\right)
$$

where

$$
s=\frac{44.5 \ln \left(9.83 / \Omega_{0} h^{2}\right)}{\sqrt{1+10\left(\Omega_{b} h^{2}\right)^{3 / 4}}} \mathrm{Mpc}
$$

approximates the sound horizon to $\sim 2 \%$ over the range $\Omega_{b} h^{2} \gtrsim 0.0125$ and $0.025 \lesssim \Omega_{0} h^{2} \lesssim 0.5$. The value of $k_{\text {peak }}$ as a function of cosmological parameters is shown in Fig. 4 .

The amplitude of the oscillations also has a non-trivial dependence on the cosmological parameters. The oscillations of course grow stronger as the baryon fraction increases. However, at fixed baryon fraction, 
(a) First Trough

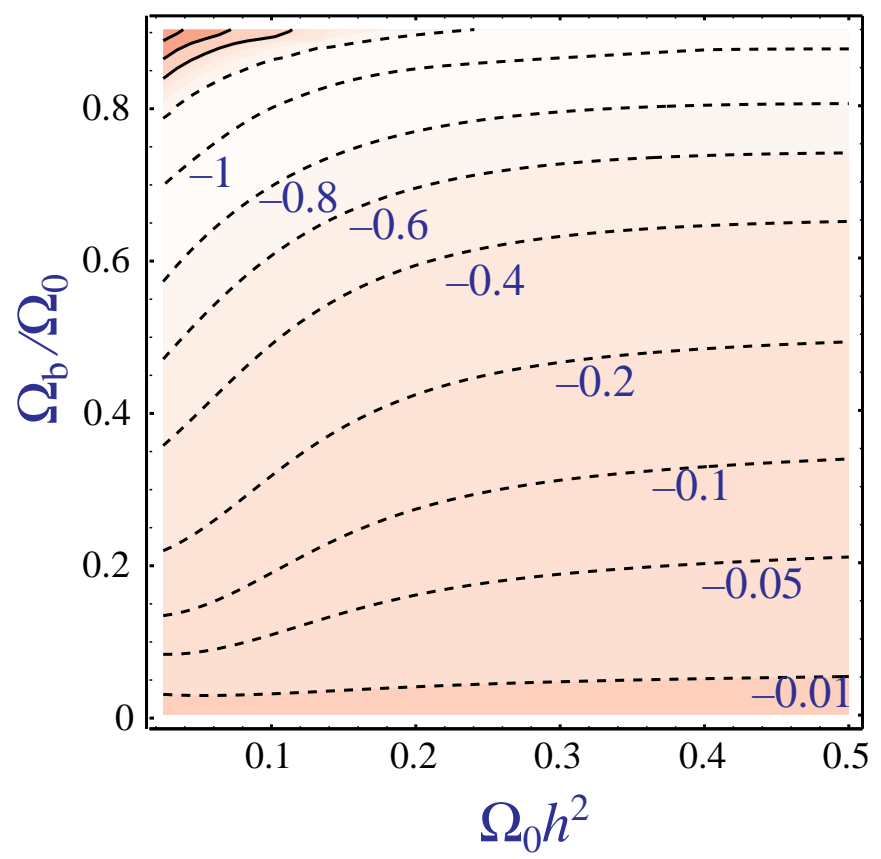

(b) First Peak

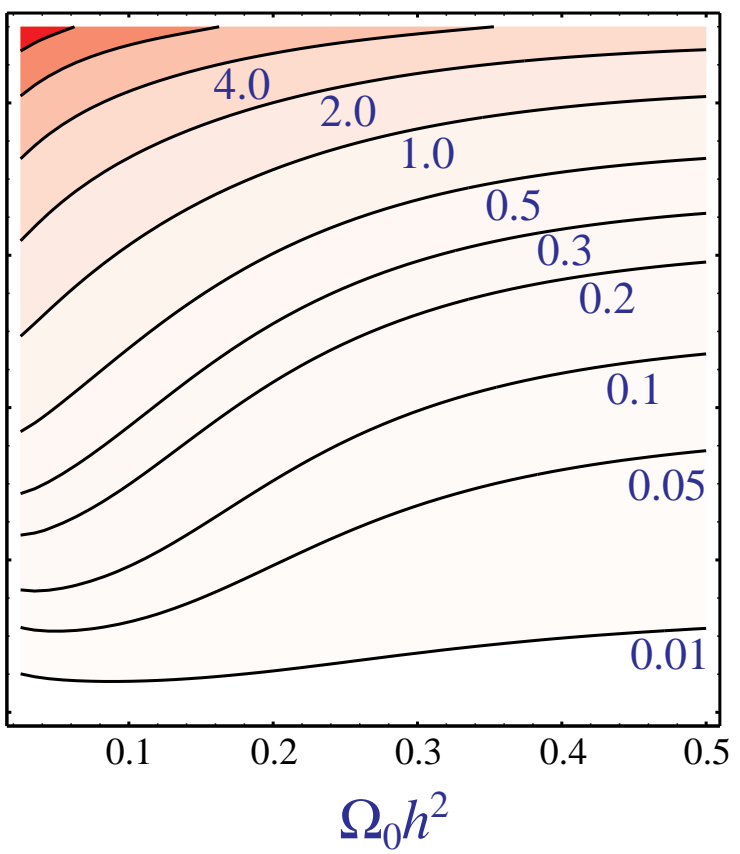

Fig. 5. - The fractional enhancement of power due to (a) the first valley and (b) the first peak relative to non-oscillatory CDM portion of the transfer function, $\left(T / T_{c}\right)^{2}-1$, at the appropriate wave vector from the fit. Unlabeled contours are at (a) -0.8, 0, 1, 3 and (b) 8.0, 16.0

they are weaker compared with CDM contributions in high $\Omega_{0} h^{2}$ due to the increase in the time available for the CDM to grow between equality and the drag epoch. While the full series of peaks and valleys may be impossible to observe due to non-linear structure formation, the first valley and peak are generally in the linear regime. In Fig. , we show the fractional enhancement of power due to the oscillations over the smooth CDM contributions. The first peak grows monotonically with the baryon fraction. The first trough is more subtle: while the transfer function at this location simply declines as the baryon fraction increases, when $T(k)$ goes negative the power, which is the square of $T$, will regenerate. Perfect cancellation of the baryon and CDM contributions occurs along the contour labeled " 1 " in Fig. 罒a; above this line the trough in amplitude becomes a peak in power as the baryon contributions come to fully dominate. A useful rough scaling as to when oscillations become important is given by

$$
\frac{\Omega_{b}}{\Omega_{0}} \gtrsim \Omega_{0} h^{2}+0.2
$$

which crudely describes the region where the change in power is greater than $\sim 20 \%$.

A related trend is the increase with $\Omega_{0} h^{2}$ of the sharpness of the decline in the baryonic sector from the knee at $k s \approx 1(T \approx 1)$ into the series of oscillations below the sound horizon. This is most easily seen in the fully baryonic models of Figure 3; near $k=0.1 \mathrm{~h} \mathrm{Mpc}^{-1}$ in the $\Omega_{0} h^{2}=0.25$ model, the transfer function drops a factor of ten in under half a decade in $k$. This break becomes even more striking in higher $\Omega_{0} h^{2}$ cases. 


\subsection{Effective Shape}

As we have seen, if $\Omega_{b} / \Omega_{0} \lesssim \Omega_{0} h^{2}+0.2$, the main effect of the baryons is not to introduce oscillations into the transfer function but to suppress the $k^{-2}$ tail from the growth of CDM perturbations. This occurs both because the CDM portion $T_{c}$ is suppressed by $\alpha_{c}$ and because the baryonic portion $T_{b}$ is providing essentially no power below the sound horizon. As noted just above, the latter transition can occur quite quickly. These suppressions indicate that the shape of the transfer function must change, with a break near the sound horizon. It is useful to quantify this effect.

Let us work forward from the zero baryon case. Here the transfer function is parameterized by $q \propto k / k_{\mathrm{eq}}$, more commonly expressed as a shape parameter $\Gamma=\Omega_{0} h$, where

$$
q=\frac{k}{h \mathrm{Mpc}^{-1}} \Theta_{2.7}^{2} / \Gamma
$$

A commonly used fitting formula to the zero-baryon limit was presented by Bardeen et al. (1986, eq. G3). However this formula fits neither the exact small-scale solution of $\$ 2.2$ nor does it have the quadratic deviation from unity required by the theory. The latter is a fundamental requirement of causality (Zel'dovich 1965), in that one power of $k$ must arise from stress gradients generating bulk velocity and a second from velocity gradients generating density perturbations. In fact the coefficient of this quadratic deviation can be calculated perturbatively if the stress gradients are dominated by the isotropic (pressure) term.

The following functional form satisfies these criteria and is a better fit to the zero baryon case extrapolated from trace-baryon models calculated by CMBfast

$$
\begin{aligned}
T_{0}(q) & =\frac{L_{0}}{L_{0}+C_{0} q^{2}}, \\
L_{0}(q) & =\ln (2 e+1.8 q), \\
C_{0}(q) & =14.2+\frac{731}{1+62.5 q} .
\end{aligned}
$$

Note that this form is not only more accurate than the Bardeen et al. (1986) one but is also simpler: there are fewer parameters and the coefficients 1.8 and 14.2 are derived theoretically. The parameter 731 , the small- $q$ quadratic deviation, has been fit rather than derived to account for the small correction due to anisotropic stress gradients. In Fig. 6 we show a comparison of this form to numerical calculations and various fitting formulae in the literature. Our formula agrees with numerical calculations at the same level as different numerical calculations agree with each other ( $\mathrm{Hu}$ et al. 1995), i.e. to $1 \%$ through the CMB and large-scale structure regimes.

The presence of baryons has commonly been included by fitting a constant shape parameter $\Gamma$ (Peacock \& Dodds 1994; Sugiyama 1995). That such an approach can work on small scales can be seen as follows. On small scales, the effect of the baryons is a constant suppression by the factor $\alpha_{c} \Omega_{c} / \Omega_{0}$. Since the transfer function there is proportional to $\left(k / \Gamma_{0}\right)^{-2}$, a rescaling of $\Gamma_{0}$ approximates this effect. However, this simple rescaling of $\Gamma_{0}$ does not properly treat the region observable through large-scale structure. Above the sound horizon, the baryons and CDM are indistinguishable, and so the transfer function is close to that parameterized by $\Gamma_{0} \equiv \Omega_{0} h$. Below the horizon, if one neglects the oscillations caused by the baryons, the transfer function is suppressed and roughly follows that of a rescaled $\Gamma$. Hence the transition around the sound horizon cannot be fit by a single $\Gamma$.

A reasonable fit to the non-oscillatory part of the transfer function can be written by rescaling $\Gamma_{\text {eff }}(k)$ 


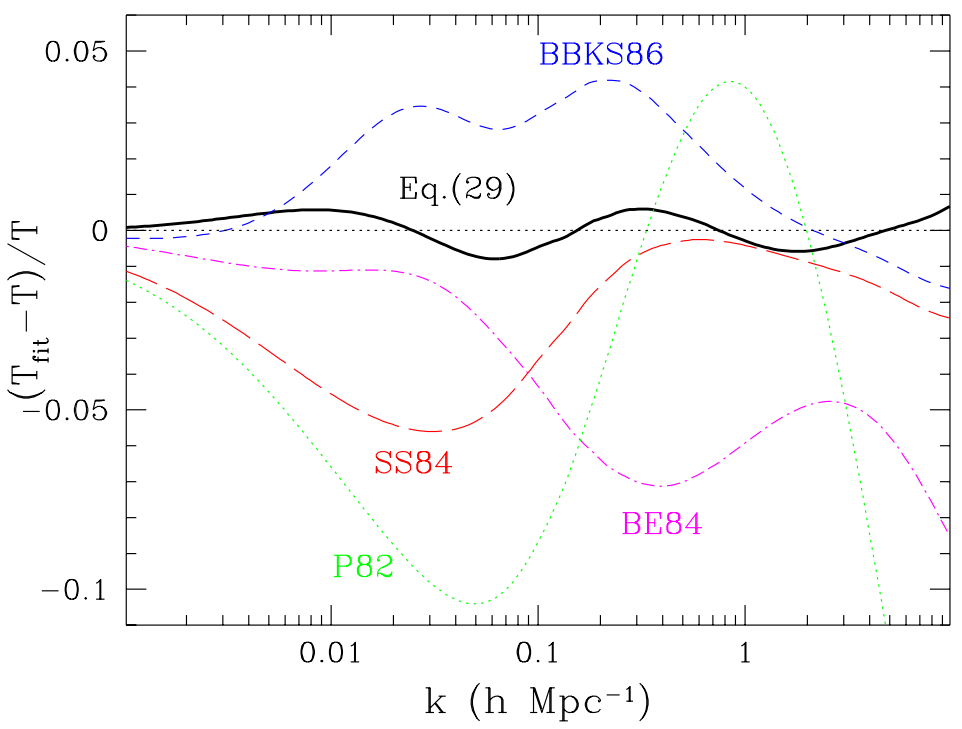

Fig. 6.- Comparison of various zero baryon transfer functions to the numerical calculation of CMBfast (Seljak \& Zaldarriaga 1996). The fitting form of equation (29) agrees with CMBfast to 1\%, whereas previous approximations Peebles (1982) [P82], Starobinsky \& Sahni (1984) [SS84], and Bardeen et al. (1986) [BBKS86]. Also shown is the form of Bond \& Efstathiou (1984) [BE84], a 3\% baryon fraction model that has often been used as a zero-baryon proxy.

as one moves through the sound horizon

$$
\begin{aligned}
\Gamma_{\mathrm{eff}}(k) & =\Omega_{0} h\left(\alpha_{\Gamma}+\frac{1-\alpha_{\Gamma}}{1+(0.43 k s)^{4}}\right), \\
\alpha_{\Gamma} & =1-0.328 \ln \left(431 \Omega_{0} h^{2}\right) \frac{\Omega_{b}}{\Omega_{0}}+0.38 \ln \left(22.3 \Omega_{0} h^{2}\right)\left(\frac{\Omega_{b}}{\Omega_{0}}\right)^{2} .
\end{aligned}
$$

Defining $q_{\mathrm{eff}}$ as in equation (28), we find that $T(k) \approx T_{0}\left(q_{\mathrm{eff}}\right)$. Figure $\mathrm{O}$ shows an example. Note the simpler form of $s$ in equation (26) may be used here. $\alpha_{\Gamma}$ is nearly $\sqrt{\alpha_{c} \Omega_{c} / \Omega_{0}}$, the radicand of which is plotted in Fig. 2; we provide the above form for simplicity and to account for small deviations at the higher $\Omega_{0} h^{2}$ values. The latter arise because the fit has been optimized in the observable region $k \lesssim 0.1 \mathrm{Mpc}^{-1}$, where the CDM transfer function is not quite in its $k^{-2}$ small-scale limit. Of course, neglecting the oscillatory contributions is not a good approximation for $\Omega_{b} / \Omega_{0} \gtrsim 0.5$. In addition to the obvious omission of the wiggles, we have fit $\alpha_{\Gamma}$ to the transfer function $T(k)$ and not the observable $T(k)^{2}$. This neglects the power introduced by the square of the oscillatory term.

We caution the reader that the small-scale asymptote $\Gamma_{\text {eff }}=\alpha_{\Gamma} \Omega_{0} h$ arises from the normalization rather than shape and therefore should not be conflated with $\Gamma$ derived from redshift surveys Peacock \& Dodds 1994). Pure normalization distinctions are not observable with current redshift surveys; instead, one estimates $\Gamma$ by fitting power spectra of arbitrary normalization and relying on the differences in shape and power-law slope introduced by the variation in $\Gamma$. However, when combined with the COBE normalization (e.g. Bunn \& White 1997), the normalized predictions from equations (30) and (31) are appropriate. 


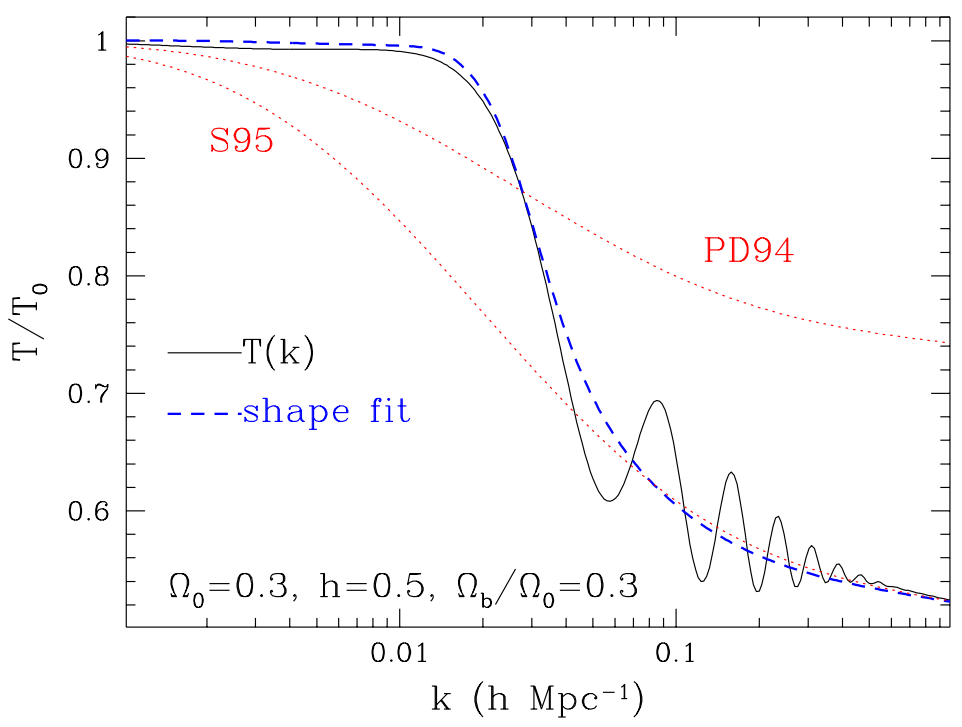

Fig. 7.- Distortions to the shape of the transfer function as measured by the true transfer function over the zero baryon form. The shape function of equation (30) adequately describes the true curve apart from the oscillations, whereas the constant shift in $\Gamma$ described by Peacock \& Dodds (1994) [PD94] and Sugiyama (1995) [S95] do not.

However, equation (30) and Fig. 7 do make clear that a simple rescaling of $\Gamma$ is not accurate near the sound horizon. Future measurements of large-scale structure expected from the Sloan Digital Sky Survey (Gunn \& Weinberg 1995) and 2-degree Field (Taylor 1995) surveys will be sufficiently precise as to detect the deviations from a single $\Gamma$ model (Tegmark 1997; Goldberg \& Straus 1997). A key signature is that the bend from $T \approx 1$ to $T \propto k^{-2}$ becomes sharper as baryons are introduced. Detecting this change will require that scales around $s / \pi$, c.f. equation (26), are well-observed.

\section{Discussion}

We have presented an accurate, well-motivated fitting form for the transfer function of a general CDMbaryon universe. The formula is generally accurate to better than $10 \%$ in fully baryonic universes and better than $5 \%$ in cosmologies with $\Omega_{b} / \Omega_{0} \lesssim 0.5$. While the available numerical codes will provide yet more accurate transfer functions, the fitting form here should be useful for characterizing trends in cosmological parameter space. Moreover, by separating the various physical aspects in the analytic form, we hope to provide physical intuition for the effects, their interrelationships, and their correspondence with analogous effects in the CMB.

As applications of the form, we gave quantitative assessments of the location and amplitude of the baryonic features in the linear regime. We quantified the suppression of power on scales below the sound horizon due to the admixture of baryons and showed that this suppression is not well-fit by a rescaling of $\Gamma$ if one is probing scales near the sound horizon. An alternative model, based on an interpolation in $\Gamma$, gives 
a reasonable fit to the non-oscillatory portion of the transfer function provided that $\Omega_{b} / \Omega_{0} \lesssim \Omega_{0} h^{2}+0.2$. Finally, we gave a new fitting form for the zero-baryon limit that is more accurate on small scales than those used previously. A summary of how to use these various formulae is given in Appendix A.

Baryonic features, like the acoustic peaks in the CMB (Hu \& White 1996), transcend the adiabatic CDM paradigm discussed in this paper. In fact any gravitational instability model where reionization took place no earlier than $z_{d} \sim 270\left(\Omega_{0} h^{2}\right)^{1 / 5}$, the Compton drag epoch for a fully ionized universe, must possess acoustic effects at some level. Evidence of these effects is strong indication that fluctuations were generated in the early universe. Moreover, when combined with CMB observations, baryonic features allow strong consistency tests for the predicted growth of fluctuations as both sets of features reflect the underlying acoustic oscillations before recombination. A measurement of the sound horizon from the matter power spectrum combined with its angular extent from the CMB would allow an angular diameter distance test for curvature in the universe that is largely free of cosmological assumptions. Baryonic features in the matter power spectrum thus represent a valuable resource for cosmological information, but one that may be difficult to mine observationally.

Acknowledgments: The CMBfast package (http://arcturus.mit.edu:80/ matiasz/CMBFAST/cmbfast.html was used to generate numerical transfer functions. We thank S. Boughn, U. Seljak, J. Silk, D. Spergel, A. Szalay, M. Tegmark, and M. White for useful discussions. W.H. acknowledges support from the W.M. Keck foundation and the hospitality of the Aspen Center for Physics. D.J.E. acknowledges support from NSF PHY-9513835.

\section{A. A User's Guide}

In this paper, we have presented a fitting form for the transfer function in a CDM and baryon cosmology with adiabatic perturbations. The formula is given as equations (2) $-(7)$, (10) $-(12)$, and (14) $-(24)$. Alternatively, if one prefers a simpler form that accurately represents the baryon-induced suppression on intermediate scales but that ignores the acoustic oscillations in the transfer function, one may use the form given in equations (26) and (28)-(31). The oscillations are fairly small ( $\lesssim 20 \%$ modulation in power) for $\Omega_{b} / \Omega_{0} \lesssim \Omega_{0} h^{2}+0.2$, so in these cases the simpler form will be appropriate for many applications. We also provide in equation (29) a more accurate form for the zero-baryon transfer function. Electronic versions of the formulae in this paper may be found at

http://www.sns.ias.edu/ whu/transfer/transfer.htm.

The power spectrum of the density fluctuations is then proportional to the initial power spectrum times the square of the transfer function. In the most usual case, the initial power spectrum is taken to be a power-law, so that $P(k) \propto k^{n} T^{2}(k)$, where $n=1$ is the familiar Harrison-Zel'dovich-Peebles scale-invariant case.

While the transfer function is independent of late-time effects such as the presence of a cosmological constant or curvature, the magnitude and time-dependence of the normalization of the power spectrum does depend on these effects. Bunn \& White (1997) calculate the present-day normalization of the power spectrum implied by the 4-year COBE anisotropy measurement and provide the following fitting forms for 
flat and open cosmologies:

$$
\begin{array}{rlr}
\left.\Delta^{2}(k)\right|_{z=0} & \equiv \frac{k^{3}}{2 \pi^{2}} P(k)=\delta_{H}^{2}\left(\frac{c k}{H_{0}}\right)^{3+n} T^{2}(k), \\
\delta_{H} & =1.95 \times 10^{-5} \Omega_{0}^{-0.35-0.19 \ln \Omega_{0}-0.17 \tilde{n}} e^{-\tilde{n}-0.14 \tilde{n}^{2}} & (\Lambda=0), \\
\delta_{H} & =1.94 \times 10^{-5} \Omega_{0}^{-0.785-0.05 \ln \Omega_{0}} e^{-0.95 \tilde{n}-0.169 \tilde{n}^{2}} \quad\left(\Lambda=1-\Omega_{0}\right),
\end{array}
$$

where $\tilde{n}=n-1$ and the contribution of tensor perturbations to the observed anisotropies has been assumed to be zero (see Bunn \& White 1997 for more details). The $1 \sigma$ statistical uncertainty is $7 \%$, and the error in the above fits are much smaller than this for $0.2 \leq \Omega_{0} \leq 1$ and $0.7 \leq n \leq 1.2$.

To extend this normalization to earlier times, one needs to scale the power spectrum by the square of the growth function $D_{1}(z)$ (Peebles 1980). As is well-known, for $\Omega_{0}=1, D_{1}=(1+z)^{-1}$ at redshift $z$. For other cosmologies, one can use the approximation (Lahav et al. 1991; Carroll et al. 1992)

$$
D_{1}(z)=(1+z)^{-1} \frac{5 \Omega(z)}{2}\left\{\Omega(z)^{4 / 7}-\Omega_{\Lambda}(z)+[1+\Omega(z) / 2]\left[1+\Omega_{\Lambda}(z) / 70\right]\right\}^{-1} .
$$

Here $\Omega(z)$ and $\Omega_{\Lambda}(z)$ are the density parameters as seen by an observer at redshift $z$; hence

$$
\begin{aligned}
\Omega(z) & =\frac{\Omega_{0}(1+z)^{3}}{\Omega_{\Lambda}+\Omega_{R}(1+z)^{2}+\Omega_{0}(1+z)^{3}}, \\
\Omega_{\Lambda}(z) & =\frac{\Omega_{\Lambda}}{\Omega_{\Lambda}+\Omega_{R}(1+z)^{2}+\Omega_{0}(1+z)^{3}},
\end{aligned}
$$

where $\Omega_{0}$ is the matter density in units of critical, $\Omega_{\Lambda}=\Lambda / 3 H_{0}^{2}$ represents the cosmological constant $\Lambda$, and $\Omega_{R}=1-\Omega_{0}-\Omega_{\Lambda}$ represents the effects of curvature.

The normalization of the power spectrum is such that the variance of mass fluctuations inside a sphere of radius $R$ is

$$
\sigma^{2}(R)=\int_{0}^{\infty} \frac{d k}{k} \Delta^{2}(k)\left(\frac{3 j_{1}(k R)}{k R}\right)^{2}
$$

where $j_{1}(x)=(x \cos x-\sin x) / x^{2}$.

\section{REFERENCES}

Bahcall, N.A. 1997, in Unsolved Problems in Astrophysics, ed. J.N. Bahcall and J.P. Ostriker, (Princeton: Princeton University Press); astro-ph/9612046

Bahcall, N.A., Fan, X., \& Cen, R. 1997, ApJ, ApJ 485, L53; astro-ph/9706018

Bardeen, J.M., Bond, J.R., Kaiser, N. \& Szalay, A.S. 1986, ApJ, 304, 15

Baugh, C.M., \& Efstathiou, G. 1993, MNRAS, 265, 145

Bennett, C. et al. 1996, ApJL, 464, L1

Bond, J.R. \& Efstathiou, G. 1984, ApJ, 285, L45

Bunn, E.F., \& White, M. 1997, ApJ, 480, 6 
Carroll, S.M., Press, W.H., Turner, E.L. 1992, ARA\&A, 30, 499

David, L.P., Jones, C., \& Forman, W. 1995, ApJ, 445, 578

Fixsen, D. et al. 1996, ApJ, 473, 576

Freedman, W.L. 1996, in Critical Dialogues in Cosmology, ed. N. Turok (Cambridge: Cambridge University Press); astro-ph/9612024

Goldberg, D.M. \& Strauss, M., 1997, preprint, astro-ph/9707209

Gunn, J.E., \& Weinberg, D.H. 1995, in Wide-Field Spectroscopy and the Distant Universe, ed. S.J. Maddox and A. Aragón-Salamanca (Singapore: World Scientific), 3

Holtzmann, J.A. 1989, ApJS, 71,1

Hu, W., Scott, D., Sugiyama, N. \& White, M. 1995, Phys. Rev. D, 52, 5498

Hu, W. \& Sugiyama, N. 1996, ApJ, 471, 542 (HS96); astro-ph/9510117

Hu, W. \& White, M. 1996, ApJ, 471, 30; astro-ph/9602019

Lahav, O., Rees, M.J., Lilje, P.B., \& Primack, J.R. 1991, MNRAS, 251, 128

Peacock, J.A. \& Dodds, S.J. 1994, MNRAS, 267, 1020.

Peebles, P.J.E. 1980, Large-Scale Structure of the Universe (Princeton: Princeton Univ. Press)

Peebles, P.J.E. 1982, ApJL, 263, L1

Peebles, P.J.E. \& Yu, J.T. 1970, ApJ, 162, 815

Press, W., \& Vishniac, E. 1980, ApJ, 236, 323

Rugers, M., \& Hogan, C.J. 1996, ApJ, 459, L1

Seljak, U. \& Zaldarriaga, M. 1996, ApJ, 469, 437; astro-ph/9603033

Silk, J. 1968, ApJ, 151, 459

Starobinsky, A.A. \& Sahni, V. 1984, in Modern Theoretical and Experimental Problems of General Relativity as quoted in Shandarin, S.F. \& Zel'dovich, Ya. B. 1984, Rev. Mod. Phys., 61, 185

Spinrad, H., Dey, A., Stern, D., Dunlop, J., Peacock, J., Jimenez, R., \& Windhorst, R. 1997, ApJ, 484, 581

Strauss, M., \& Willick, J. 1995, Phys. Rep., 261, 271

Sugiyama, N. 1995, ApJS, 100, 281; astro-ph/9412025

Sunyaev, R., \& Zel'dovich, Ya. B. 1970, Ap\&SS, 7, 3

Taylor, K. 1995, in Wide-Field Spectroscopy and the Distant Universe, ed. S.J. Maddox and A. AragónSalamanca (Singapore: World Scientific), 15

Tegmark, M., 1997, preprint, astro-ph/9706198

Tytler, D., Fan, X.M., \& Burles, S. 1996, Nature, 381, 207 
Walker, T.P., Steigman, G., Kang, H., Schramm, D.M., Olive, K.A. 1991, ApJ, 376, 51

Weinberg, D.H., Miralda-Escudé, J., Hernquist, L., \& Katz, N. 1997, submitted to ApJ; astro-ph/9701012

White, S.D.M., Navarro, J.F., Evrard, A.E., \& Frenk, C.S. 1993, Nature, 366, 429

Zel'dovich, Ya. B. 1965, Adv. Astron. Astrophys., 3, 241 